\title{
Catheter Related Bloodstream Infection by Brevibacterium casei in a Patient with B Cell Acute Lymphoid leukemia - A Case Report
}

\author{
Smita Deshkar ${ }^{1}$, Niranjan Patil ${ }^{2}$, Pranali Balmiki ${ }^{3}$ \\ ${ }^{1}$ Microbiologist, ${ }^{2}$ HOD Infectious Diseases Department, ${ }^{3}$ Deputy Section Head, Microbiology, \\ Metropolis Healthcare Limited, Global Reference Laboratory, Mumbai
}

Corresponding Author: Smita Deshkar

\begin{abstract}
Patients with long term indwelling catheters with underlying immunosuppression or comorbid conditions are predisposed to develop catheter related blood stream infections with unusual organisms. Brevibacterium spp. are catalase-positive, non spore-forming, nonmotile, aerobic, Gram-positive bacteria. Brevibacterium spp were not considered a human pathogen, until recently few infections were noted. We report a case of catheter related blood stream infection by Brevibacterium casei in 17 year old young adult with B cell acute lymphoid leukemia. Patient was treated successfully with intravenous Vancomycin and Piperacillin-tazobactam along with peripherally inserted central catheter removal.
\end{abstract}

Keywords: Brevibacterium casei, Catheter related blood stream infections, Sepsis, Immunocompromised

\section{INTRODUCTION}

Indwelling catheter related blood stream infections (CRBSI) pose a massive challenge, especially in the management of patients who are immunocompromised or receiving cancer chemotherapy. Chemotherapy induces long-term neutropenia, which greatly increases the risk of infection. [1] Although the spectrum of possible pathogens from CRBSIs in clinical settings is rapidly growing and the most common organisms isolated are coagulasenegative Staphylococci and Staphylococcus aureus. ${ }^{[1]}$ Brevibacterium spp. was not considered as human pathogens, until recently few infections were noted in immunocompromised patient. Literature regarding the infection is also sparse, so we found it worthwhile to report this case of CRBSI and sepsis with Brevibacterium casei in patient with acute lymphoid leukemia (ALL).

\section{CASE REPORT}

A 17 years old male patient with Bcell ALL was admitted with history of high grade fever since one day. He had received bone marrow transplant 6 months before. Combination chemotherapy was given and complete remission was achieved. Patient was on maintenance chemotherapy with CNS prophylaxis. Patient had developed fever with chills 9 days after maintenance therapy. He appeared toxic, with temperature $102.8^{0} \mathrm{~F}$, pulse rate $110 / \mathrm{min}$, respiratory rate $26 / \mathrm{min}$ and blood pressure 90/60 mm Hg. Systemic examination was unremarkable.

The complete blood cell count (CBC) at the time of fever included a white blood cell count of $1783 \mathrm{~mm}^{3} / \mathrm{L}$ with absolute neutrophil count of $387 \mathrm{~mm}^{3} / \mathrm{L}$, haemoglobin of $7.17 \mathrm{~g} / \mathrm{dL}$ and a platelet count of $55 \times 10^{3} \mathrm{~mm}^{3} / \mathrm{L}$. C-reactive protein was $9.1 \mathrm{mg} / \mathrm{dL}$. Renal function tests and urine routine examination did not show any significant abnormality. Two sets of blood cultures were taken from the peripheral vein and peripherally inserted central catheter 
(PICC) line. The PICC was removed on the day of hospital admission. As his whole blood cell counts revealed pancytopenia; he was then put on antibiotic Piperacillintazobactam empirically.

After 24 hours of incubation in automated blood culture system (BD BACTEC TM FX Instrument, Becton Dickinson, USA), blood culture bottles flagged positive for growth. The differential time to positivity (DTP) between blood taken from PICC and the peripheral vein was 5 hours 20 minutes. The Gram stain smears from blood culture bottles showed Gram positive, slender, slightly curved, rod shaped bacteria. After 24 hours of incubation at $37^{\circ} \mathrm{C}$ in a $\mathrm{CO}_{2}$ atmosphere, on sheep blood agar colonies were grayishwhite in colour, non-haemolytic, smooth, round and had a distinctive cheese odour (Figure 1). No colony growth was observed on MacConkey agar. On nutrient agar, colonies were small, opaque, convex, with a shiny, smooth surface. After 4-7 days of incubation the colonies became large, 2-4 $\mathrm{mm}$ in diameter. (Figure 2) The isolate was catalase positive, oxidase negative and nonmotile. On further biochemical testing, glucose was oxidized, urea was not hydrolysed, nitrates were reduced to nitrites, esculin and gelatin hydrolysis was positive. It was presumptively identified as Brevibacterium spp. Subsequently, it was identified as Brevibacterium casei (B. casei) by Matrix-Assisted Laser Desorption/Ionization-Time of Flight Mass Spectrometry (MALDI TOF MS) (bioMérieux, France) with 99. 9 confidence value. Diagnosis of CRBSI caused by $B$. casei was made. Vancomycin was then added to the treatment protocol.

Antimicrobial susceptibility testing was done and interpreted as per Clinical and Laboratory Standards Institute (CLSI) M45 recommendations for Corynebacterium spp. ${ }^{[2]}$ The isolate was found to be susceptible to Vancomycin (MIC 0.25 $\mu \mathrm{g} / \mathrm{mL}$ ), Meropenem (MIC $0.5 \mu \mathrm{g} / \mathrm{mL}$ ), Cefepime (MIC $1 \mu \mathrm{g} / \mathrm{mL}$ ) and Gentamicin (MIC $2 \mu \mathrm{g} / \mathrm{mL}$ ). Resistance was noted for
Penicillin (MIC $\geq 8 \mu \mathrm{g} / \mathrm{mL}$ ) Cefotaxime (MIC $\geq 4 \mu \mathrm{g} / \mathrm{mL}$ ), Clindamycin (MIC $\geq 4$ $\mu \mathrm{g} / \mathrm{mL}$ ) Ciprofloxacin (MIC $\geq 4 \mu \mathrm{g} / \mathrm{mL}$ ) and intermediate susceptibility was observed for Erythromycin (MIC $1 \mu \mathrm{g} / \mathrm{mL}$ ). Fever subsided after 24 hours of antibiotic therapy. Two follow-up blood cultures were collected in the subsequent weeks which were negative for any bacterial growth. The patient recovered 10 days after starting therapy. Bacteraemia due to the B. case $i$ had not recurred for more than six months.

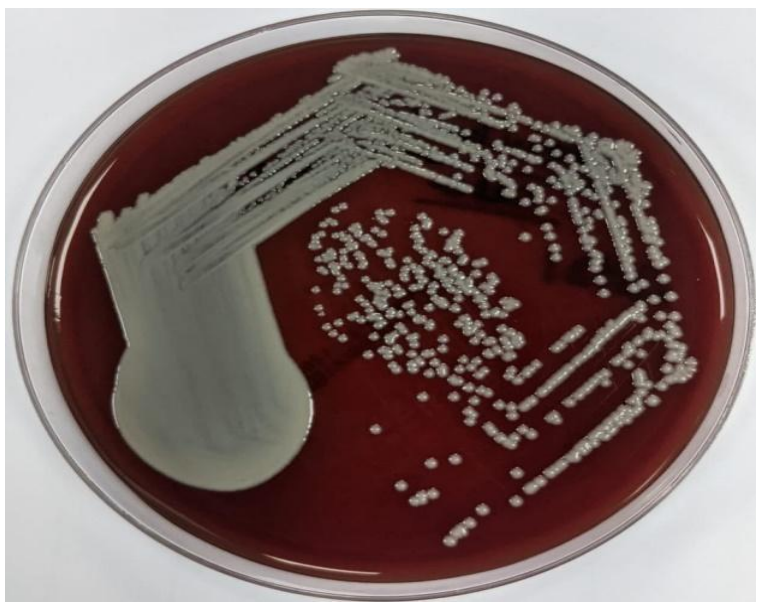

Figure 1: Grayish-white, non-haemolytic, smooth colonies $B$. casei on a sheep blood agar

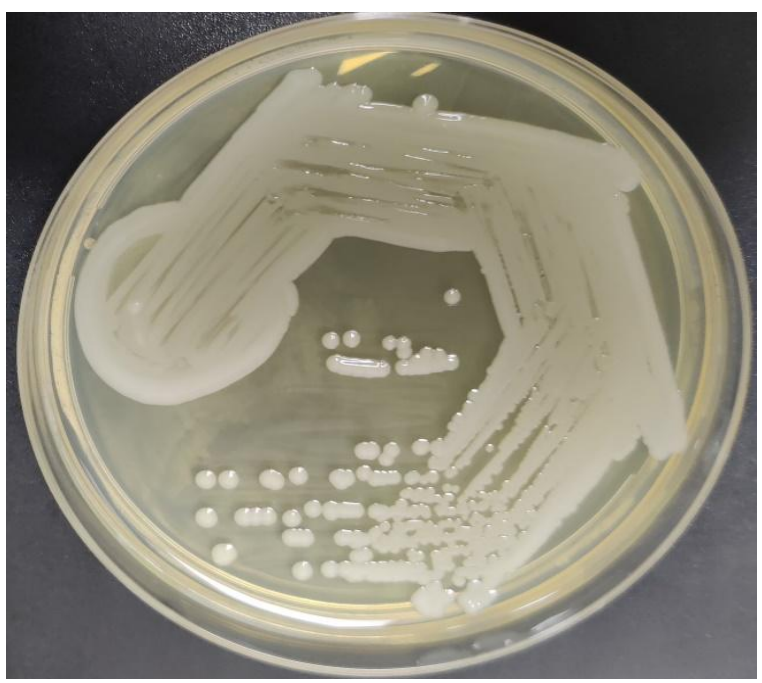

Figure 2: Opaque, convex, shiny, smooth colonies $B$. casei on a nutrient agar

\section{DISCUSSION}

The genus Brevibacterium was established in 1953 by Breed and is characterized by non-sporing, non-motile, catalase-positive, Gram-positive rods. ${ }^{[3]}$ In nature, Brevibacterium contributes notably 
to the aroma and colour (orange pigment) of surface-ripened cheese. The organism can also be found in raw milk and human skin. [4] Presently, the genus Brevibacterium consists of 45 different species, of which only nine, namely, B. linens, B. casei, $B$. epidermidis, B. iodinum, B. mcbrellneri, $B$. otitidis, B. paucivorans, B. sanguinis and the recently described $B$. massiliense have been isolated from clinical samples. Not only $B$. case $i$ is by far the most frequently isolated Brevibacterium species from otherwise sterile human sites but also opportunistic infections by $B$. casei mostly in nosocomial settings, are on the rise. ${ }^{[4]}$ Reports of Brevibacterium causing a variety of infections like bacteraemia with sepsis, brain abscess, peritonitis and endocarditis have been documented. ${ }^{[1,5-9]}$ Most patients had presented with specific underlying conditions such as malignant tumours, renal failure or an immunocompromised status. [1, 7-9] However, Kumar VA et al ${ }^{[4]}$ and Ulrich $\mathrm{S}$ et al ${ }^{[10]}$ had reported $B$. casei infection in immunocompetent patients. Long term medical catheters are often required for treatment in patients with underlying diseases such as malignant tumours, renal failure or an immunocompromised status. These indwelling catheters increase the risk of acquiring CRBSIs. Catheter related infections are reported in $7-33 \%$ cases, secondary to either chronic colonization of the intravascular portion of the catheter from the exit site or external portions of the catheter. [11] In general, management of CRBSI includes systemic antibiotic therapy, choice and duration of therapy depending upon clinical symptoms and underlying disease along with catheter removal or replacement. In cases reported in literature, Vancomycin, Ceftazidime, Ciprofloxacin and Piperacillin-tazobactam were preferred treatment options. (Table 1) No relapsed infections had been noted in patients with CRBSI due to B. case i in whom catheter removal was performed as an empiric therapy. [7-10] However, there is no consensus about the management of uncomplicated CRBSI. Antibiotic-lock has been proposed for cases of catheter-related bacteraemia caused by Staphylococcus aureus, coagulase-negative Staphylococci and Gram-negative bacilli. ${ }^{[12]}$

Table 1: Characteristics, treatment and outcomes of CRBSI by B. casei

\begin{tabular}{|c|c|c|c|c|c|c|}
\hline $\begin{array}{l}\text { Study } \\
\text { (Year) }\end{array}$ & $\begin{array}{c}\text { Age } \\
\text { (Years) } \\
\text { Gender }\end{array}$ & $\begin{array}{l}\text { Underlying } \\
\text { Conditions }\end{array}$ & $\begin{array}{c}\text { Clinical } \\
\text { Presentation }\end{array}$ & Device & Empiric Therapy & $\begin{array}{l}\text { Treatment After } \\
\text { Relapse }\end{array}$ \\
\hline $\begin{array}{l}\text { Ochi F et al } \\
(2021)\end{array}$ & $8, \mathrm{~F}$ & AML, FN & CRBSI & PICC & $\begin{array}{c}\text { MERO +VAN+device } \\
\text { removal }\end{array}$ & No relapse \\
\hline $\begin{array}{l}\text { Bal ZS et al }{ }^{[7]} \\
(2015)\end{array}$ & $6, \mathrm{M}$ & ALL, FN & CRBSI & $\begin{array}{l}\text { Hickman } \\
\text { Catheter }\end{array}$ & PIP/ TAZ+VAN & No relapse \\
\hline $\begin{array}{l}\text { Magi B et al } \\
(2018)\end{array}$ & $48, \mathrm{~F}$ & $\begin{array}{l}\text { Breast } \\
\text { cancer }\end{array}$ & CRBSI & $\begin{array}{l}\text { Port a } \\
\text { cath }\end{array}$ & $\begin{array}{c}\text { CIP + TEIC + } \\
\text { device removal, LZD }\end{array}$ & No relapse \\
\hline $\begin{array}{l}\text { Janda W et al }{ }^{[9]} \\
(2003)\end{array}$ & $34, \mathrm{M}$ & AIDS & $\begin{array}{l}\text { CRBSI } \\
+ \text { Sepsis }\end{array}$ & $\begin{array}{l}\text { Hickman } \\
\text { Catheter }\end{array}$ & $\begin{array}{c}\mathrm{CAZ}+\mathrm{VAN}+\text { device } \\
\text { removal }\end{array}$ & No Relapse \\
\hline $\begin{array}{l}\text { Ulrich S et al }{ }^{[10]} \\
(2006)\end{array}$ & $62, \mathrm{~F}$ & $\mathrm{PH}$ & $\begin{array}{l}\text { CRBSI } \\
+ \text { Sepsis }\end{array}$ & $\mathrm{CVC}$ & $\begin{array}{c}\text { MFLX +VAN + } \\
\text { device removal }\end{array}$ & No relapse \\
\hline $\begin{array}{l}\text { Beukinga I et al } \\
{ }^{[13]}(2004)\end{array}$ & $43, \mathrm{~F}$ & $\begin{array}{l}\text { Chron's } \\
\text { Disease }\end{array}$ & CRBSI & $\begin{array}{l}\text { Port a } \\
\text { cath }\end{array}$ & VAN & $\begin{array}{c}\text { AMC, MERO, VAN + } \\
\text { device removal }\end{array}$ \\
\hline
\end{tabular}

AIDS - acquired immunodeficiency syndrome; ALL - acute lymphoblastic leukaemia; AMC- Amoxycilin/ Clavulanic acid AML - acute myeloid leukaemia; CAZ - ceftazidime; CIP - ciprofloxacin; CRBSI - catheter related blood stream infection; CVC - central venous catheter; F - female; M -male; MERO - meropenem; MFLX, - moxifloxacin; FN- febrile neutropenia; NHL- Non Hodgkin's lymphoma; PICC - peripherally inserted central catheter; PH - pulmonary hypertension; PIP/ TAZ - piperacillin- tazobactam; VAN - vancomycin.

Our patient had presented with B cell ALL with long term indwelling PICC. He had developed CRBSI with DTP of more than five hours between PICC and peripheral vein blood culture. Species level identification was done by MALDI TOF
MS (bioMérieux, France). MALDI-TOF MS relies on measuring microbial proteins that are typically well conserved within a species. Thus, it provides a more reliable means of discriminating one species from another with a high degree of confidence. 
[14] The turnaround time with which MALDI-TOF MS can identify microorganisms helps to quickly guide treatment decisions, which is especially critical when the infecting pathogen is unexpected like in our case. This helps in reduction in the length of hospitalization as well. Although exact virulence factors and pathogenesis of $B$. casei infection are not known, neutropenia related to chemotherapy with abrogated immune responses likely contributed to infection, with likely portal of entry being the compromised mucosal integrity secondary to PICC. We could not establish the exact source of infection as cultures of intravenous fluids and chemotherapeutic drugs infused to patients were sterile. There is no standardised treatment for B. casei. Additionally, B. case $i$ isolates are known to exhibit varying degrees of susceptibility to a variety of antimicrobial agents. [1] Through extrapolation of the CLSI M45 criteria for MIC breakpoints for Corynebacterium spp., our isolate was found susceptible glycopeptides, carbapenems, fourth generation cephalosporins and aminoglycosides. Patient was treated with combination therapy of Piperacillintazobactam and Vancomycin to which he responded well. Recurrence of bacteraemia caused by the same strain of B. casei had been demonstrated up to 5 months following initial adequate therapy. ${ }^{[13]}$ Based on this, it is suggested that catheter removal should be the preferred treatment for associated $B$. casei bloodstream infection. ${ }^{[13]}$ In our patient, PICC was removed on day of hospital admission, which helped along with antibiotic therapy to achieve infection source control and to reduce bio burden.

\section{CONCLUSION}

$B$ casei is able to cause infection in patients with profound immunosuppression. Malignancies with prolonged neutropenia and long term indwelling catheters act as an independent risk factors for bacteraemia with $B$ casei. Identification may be difficult by conventional methods only; combination of conventional and automated methods can give correct species level identification. It is of utmost importance to perform antimicrobial susceptibility testing due to its varying degrees of susceptibility to various antimicrobial agents. Multicentre studies should be done to establish clinical breakpoints for $B$ case $i$.

\section{Acknowledgement: None}

\section{Conflict of Interest: None}

\section{Source of Funding: None}

\section{REFERENCES}

1. Ochi F, Tauchi H, Moritani K, Murakami S, Miyamoto H, Ueda M, et al. A CatheterRelated Bloodstream Infection by Brevibacterium casei in a child with acute myeloid leukemia: Case Report and Literature Review. Case Rep Pediatr 2021; 2021: 6691569.

2. Clinical and Laboratory Standards Institute. Methods for antimicrobial dilution and disk susceptibility testing of infrequently isolated and fastidious bacteria. $3^{\text {rd }}$ ed. CLSI guidelines M45. Wayne PA: Clinical and Laboratory Standards Institute; 2015.

3. Breed RS. The families developed from Bacteniaceae Cohn with a description of the family Brevibacteriaceae. Riass. Commun. VI Congr. Int. Microbiol 1953; 1:10-15.

4. Kumar VA, Augustine D, Panikar D, Nandakumar A, Dinesh KR, Karim S, et al. Brevibacterium casei as a cause of brain abscess in an immunocompetent patient. J Clin Microbiol 2011;49 (12):4374-6.

5. Wauters G, Van Bosterhaut B, Avesani V, Cuvelier R, Charlier J, Janssens M, et al. Peritonitis due to Brevibacterium otitidis in a patient undergoing continuous ambulatory peritoneal dialysis. J Clin Microbiol 2000; 38: 4292-4293.

6. Dass KN, Smith MA, Gill VJ, Goldstein SA, Lucey DR. Brevibacterium endocarditis: a first report. Clin Infect Dis 2002; 35: e20-e21.

7. Bal ZS, Sen S, Karapinar DY, Aydemir S, Vardar F. The first reported catheter-related Brevibacterium casei bloodstream infection in a child with acute leukemia and review of 
the literature. 5e Braz J Infect Dis 2015; 19(2): 213-5.

8. Magi B, Migliorini L, Sansoni A, Cusi MG. Brevibacterium casei bacteraemia in a porta-cath carrier patient: a case report. Le Infezioni in Medicina 2018;26(3):263-5.

9. Janda W, Tipirneni P, Novak RM. Brevibacterium casei bacteraemia and line sepsis in a patient with AIDS. J Infect 2003; 46(1):61-4.

10. Ulrich S, Zbinden R, Pagano M, Fischler M, Speich R. Central venous catheter infection with Brevibacterium sp. in an immunocompetent woman: case report and review of the literature. Infection 2006; 34(2);103-6.

11. Galloway S, Bodenham A, Long-term central venous access, BJA: British Journal of Anaesthesia 2004;92(5) Volume 92(5): 722-34.

12. Mermel LA, Farr BM, Sherertz RJ, Raad II, O'Grady N, Harris JS, et al. Infectious Diseases Society of America; American College of Critical Care Medicine; Society for Healthcare Epidemiology of America. Guidelines for the management of intravascular catheter-related infections. Infect Control Hosp Epidemiol. 2001; 22 (4):222-42.

13. Beukinga I, Rodriguez-Villalobos $\mathrm{H}$, Deplano A, Jacobs F, Struelens MJ. Management of long-term catheter related Brevibacterium bacteraemia. Clin Microbiol Infect. 2004; 10(5):465-7.

14. Rychert J. Commentary: Benefits and Limitations of MALDI-TOF Mass Spectrometry for the Identification of Microorganisms. J Infectiology. 2019; 2(4): $1-5$.

How to cite this article: Deshkar S, Patil N, Balmiki P. Catheter related bloodstream infection by Brevibacterium casei in a patient with $\mathrm{b}$ cell acute lymphoid leukemia-a case report. International Journal of Research and Review. 2021; 8(7): 9-13. DOI: https://doi.org/ 10.52403/ijrr.20210702 